\title{
From Display to Labelled Proofs for Tense Logics ${ }^{\star}$
}

\author{
Agata Ciabattoni, Tim Lyon, and Revantha Ramanayake \\ Institut für Computersprachen, Technische Universität Wien, 1040 Wien, Austria \\ agata@logic.at, lyon@logic.at, revantha@logic.at
}

\begin{abstract}
We introduce an effective translation from proofs in the display calculus to proofs in the labelled calculus in the context of tense logics. We identify the labelled calculus proofs in the image of this translation as those built from labelled sequents whose underlying directed graph possesses certain properties. For the basic normal tense logic Kt, the image is shown to be the set of all proofs in the labelled calculus G3Kt.
\end{abstract}

Keywords: display calculus, labelled calculus, structural proof theory, tense logic, modal logic

\section{Introduction}

The widespread application of logical methods in several areas of computer science, epistemology, and artificial intelligence has resulted in an explosion of new logics - each requiring an analytic proof calculus to facilitate study and applications. The reason is that the rules in an analytic calculus (de)compose the formula to be proved in a stepwise manner. This systematic decomposition can be exploited to prove important metalogical properties of the formalized logics and is central to developing automated reasoning methods. Being relatively simple and not requiring much technical machinery ('bureaucracy'), the sequent calculus has always been the most popular formalism to use and try to construct analytic calculi. However, its simplicity means that it is also limited in its expressive power, and is hence unable to support analytic calculi for the many logics of interest. This has motivated the search for other, more expressive formalisms. Many proof formalisms generalizing the sequent calculus have been introduced in the last 30 years; each of them incorporates the bureaucracy in a distinct way and hence possesses distinct strengths, weaknesses, and expressive power. In particular, certain formalisms are more helpful than others for proving certain computational or metalogical properties. For this reason, it is fruitful to study logics in a number of different formalisms. For example, a large class of extensions of the minimal tense logic $\mathrm{Kt}$ have been presented as instances of

\footnotetext{
* This is a pre-print of an article published in Logical Foundations of Computer Science. The final authenticated version is available online at: https://doi.org/10. 1007/978-3-319-72056-2_8
} 
the labelled calculus (e.g., [21/17]) and of the display calculus [1422/10]. The former is an extension of the sequent calculus in which the relational semantics of the formalized logics is made an explicit part of the syntax; the latter extends Gentzen's language of sequents with new structural connectives that allow each formula in a sequent to be "displayed" as the whole of the antecedent or the whole of the succedent.

Labelled and display calculi substantially differ in their nature. Display calculi are typically internal in the sense that each step in a proof can be read as a formula of the $\operatorname{logic}{ }^{1}$ In general, labelled calculi appear to manipulate formulae from a more expressive language which partially encodes the logic's semantics, and are hence termed external. Internal and external calculi have been introduced and studied within two essentially independent-and sometimes competing-streams in proof theory. These calculi possess different properties and lead to distinct proofs.

An effective way to relate calculi is by defining embeddings, i.e. functions that stepwise transform any proof in a calculus into a proof of the same formula in another calculus. A crucial feature of such a function is that the structural properties of the derivation are preserved in the translation. Such embeddings permit the transfer of certain proof theoretic results, thus alleviating the need for independent proofs in each system (see 911118]). Moreover they shed light on the role of bureaucracy in proof calculi, and on the relationships between different syntactic and semantic presentations of a logic.

In this paper we investigate the relationships between display and labelled proofs for a well known class of tense logics obtained by extending $\mathrm{Kt}$ with Scott-Lemmon [15] axioms $\nabla^{h} \square^{i} p \rightarrow \square^{j} \diamond^{k} p(h, i, j, k \geq 0)$. This class is an adequate case study as it includes many interesting/well-known logics, its display calculi are all internal, and the display and labelled rules capturing the Scott-Lemmon axiom $\$^{2}$ have a simple form. Due to their distinct foundational origins-the algebraic semantics for display calculi [14 and Kripke semantics for labelled calculi [17]-the relationship between their proofs is prima facie unclear; this is particularly true for the direction from labelled to display proofs (e.g., 19] contains a translation of display sequents into labelled sequents).

Exploiting the work of Goré et al. [10] who present the display calculus for the basic tense logic $\mathrm{Kt}$ as a nested sequent with two types of nesting constructors, we show the equivalence of the display calculus to a calculus on labelled directed graphs whose underlying undirected graph is a tree. These structures -labelled UT graphs-are a natural generalization of the labelled trees shown in 11] to correspond to nested sequents [3]13.

In particular, we give a bi-directional embedding between proofs in the display calculus and the labelled UT graph calculus. The latter are then mapped into Negri's [17 labelled sequent proofs. In the reverse direction, we then consider

\footnotetext{
${ }^{1}$ More specifically, this is true of a display calculus for a logic such that every structural connective can be interpreted as a connective of the logic.

${ }^{2}$ Extending to primitive tense axioms [14] is straightforward though more syntactically involved.
} 
specifically Negri's labelled calculus for Kt and show that every derivation there is a derivation in the labelled UT graph calculus.

\section{Display and Labelled Calculi for Tense Logics}

The tense logic Kt extends the normal modal logic $\mathrm{K}$ with the tense connectives and $\boldsymbol{\square}$ and the following axioms and inference rule (see, e.g. [24]):

$$
\begin{aligned}
& \mathbf{\square}(p \rightarrow q) \rightarrow\left(\mathbf{\square}_{p} \rightarrow \mathbf{\square}_{q}\right) \quad \diamond p \rightarrow \neg \neg p \quad \frac{A}{\mathbf{\square} A}(\mathrm{nec}) \\
& p \rightarrow \square \vee p \quad p \rightarrow \square \diamond p
\end{aligned}
$$

An intuitive interpretation of $\square A$ is the statement "it will always be the case that $A$ " (i.e. it is necessarily the case that in the future $A$ ). Then $A$ can be interpreted as "it has always been the case that $A$ " (it is necessarily the case that in the past $A$ ). Then $\diamond A$ may be interpreted as "it is possible that in the future $A$ ", and $A$ as "it is possible that in the past $A$ ". Of course, suitable other interpretations may be used as demanded by application.

We assume that our language consists of formulae in negation normal form, where all negation signs are pushed inward onto the propositional atoms. In particular, formulae are built from literals $p$ and $\bar{p}$ using $\wedge, \vee, \diamond, \square, \bullet$, and $\boldsymbol{\square}$. Note that all results still hold for the full language where the $\neg, \rightarrow$, and $\leftrightarrow$ connectives are taken as primitive. Nevertheless, we restrict ourselves to negation normal form for matters of convenience.

The logics we consider in this paper are extensions of Kt with the ScottLemmon axioms $\diamond^{h} \square^{i} p \rightarrow \square^{j} \diamond^{k} p$ (or equivalently, $\diamond^{h} \diamond^{j} p \rightarrow \diamond^{i} \diamond^{k} p$ ), for $h, j, i, k \geq 0$. In negation normal form and in the absence of implication, the axioms become $\square^{h} \diamond^{i} \bar{p} \vee \square^{j} \diamond^{k} p$ (equivalently, $\square^{h} \square^{j} \bar{p} \vee{ }^{i} \diamond^{k} p$ ). We have limited ourselves here to the Scott-Lemmon axioms in order to simplify the notation and exposition, and also because this class of axioms is well-known within the modal logic community. Nevertheless, it is worth observing that our results extend in a natural way beyond the Scott-Lemmon axioms to Kracht's 14 primitive tense axioms (or, equivalently, $\mathcal{I}_{2}[5]$ or analytic inductive [12] axioms).

\subsection{Display Calculi for Tense Logics}

Introduced under the name Display Logic, Belnap's Display Calculus [1] generalises Gentzen's sequent calculus by supplementing the structural connective (comma) with new structural connectives. The beauty of the display calculus lies in a general cut-elimination theorem for all calculi obeying eight easily verifiable syntactic conditions 122; this makes the display calculus a good candidate for capturing large classes of logics in a unified way, irrespective of their semantics or connectives.

We will present Goré et al.'s [10 display calculus SKT for Kt. This calculus can be seen as a one-sided version of Kracht's [14 display calculus for Kt, and also as a variant of Kashima's calculus [13. The sequents of SKT are generated by the following grammar: $X:=A|X, X| \circ\{X\} \mid \bullet\{X\}$. 
Definition 1 (The Calculus SKT[10]).

$$
\begin{gathered}
\frac{\Gamma, A, B}{\Gamma, p, \bar{p}}(\text { id }) \quad \frac{\Gamma, A}{\Gamma, A \vee B}(\vee) \quad \frac{\Gamma, B}{\Gamma, A \wedge B}(\wedge) \\
\frac{\Gamma, \Delta, \Delta}{\Gamma, \Delta}(\mathrm{ctr}) \quad \frac{\Gamma}{\Gamma, \Delta}(w k) \quad \frac{\Gamma, \circ\{\Delta\}}{\bullet\{\Gamma\}, \Delta}(r f) \frac{\Gamma, \bullet\{\Delta\}}{\circ\{\Gamma\}, \Delta}(r p) \\
\frac{\Gamma, \bullet\{A\}}{\Gamma, \square A}(\mathbf{\square}) \quad \frac{\Gamma, \circ\{A\}}{\Gamma, \square A}(\square) \quad \frac{\Gamma, \bullet\{\Delta, A\}, \bullet A}{\Gamma, \bullet\{\Delta\}, \bullet A}(\bullet) \frac{\Gamma, \circ\{\Delta, A\}, \diamond A}{\Gamma, \circ\{\Delta\}, \diamond A}(\diamond)
\end{gathered}
$$

SKT is referred to as a shallow nested sequent calculus because (i) the $\circ\{\}$ and - \{\} provide (two types of) nesting and (ii) all the rules are shallow in the sense that they operate at the root of the sequent (when the sequent is viewed in terms of its grammar tree). Although the rules in SKT are shallow, the two rules (rf) and (rp) can be used to bring nested formulae to the root.

Definition 2 (display property). A display calculus has the display property if it contains a set of rules (the 'display rules') such that for any sequent $X$ containing an occurrence of $Y$, there exists $Z$ such that $Y, Z$ is derivable from $X$ using the display rules.

The display property states that any substructure in $X$ can be brought to the 'top level' using the display rules. By inspection, SKT has the display property when $\{(\mathrm{rp}),(\mathrm{rf})\}$ is chosen to be the set of display rules. Incidentally, the display property is a crucial component in the proof of the general cut-elimination theorem. The interpretation $\mathcal{I}$ of a display sequent as a tense formula is defined as follows.

$$
\begin{array}{ll}
\mathcal{I}(A)=A \text { for every formula } A & \mathcal{I}(\circ X)=\square \mathcal{I}(X) \\
\mathcal{I}(X, Y)=\mathcal{I}(X) \vee \mathcal{I}(Y) & \mathcal{I}(\bullet X)=\square \mathcal{I}(X)
\end{array}
$$

A modular method of extending a base display calculus for $\mathrm{Kt}$ by a large class of axioms inclusive of the Scott-Lemmon axioms was introduced in 14] (see also [5]). Following [14, Goré et al. [10] present the rule $d(h, i, j, k)$ corresponding to the Scott-Lemmon axiom $\mathbf{\square}^{h} \square^{j} \bar{p} \vee \diamond^{i}{ }^{k} p$.

$$
\frac{\Gamma, \circ^{i}\left\{\bullet^{k}\{\Delta\}\right\}}{\Gamma, \bullet^{h}\left\{o^{j}\{\Delta\}\right\}} d(h, i, j, k)
$$

Theorem 1 ([14 10]). Let $S$ be any finite set of Scott-Lemmon axioms. $A \in$ $K t+S$ iff $A$ is derivable in $S K T+S^{\prime}$, where $S^{\prime}=\left\{d(h, i, j, k) \mid \boldsymbol{\square}^{h} \square^{j} \bar{p} \vee \diamond^{i}{ }^{k} p \in\right.$ $S\}$.

\subsection{Labelled Calculi for Tense Logics}

Labelled sequents 816] generalise Gentzen sequents by the prefixing of state variables to formulae occurring in the sequent and by making the relational semantics explicit in the syntax. A labelled sequent has the form $\mathcal{R}, \Gamma$ where the relation mset (multiset) $\mathcal{R}$ consists of terms of the form $R x y$. Meanwhile $\Gamma$ is a 
multiset of labelled formulae (e.g. $x: A \rightarrow B, y: p$ ). A labelled sequent can be viewed as a directed graph (defined using the set $\mathcal{R}$ ) with formulae decorating each node [19|20].

Negri [17] has presented a method for generating cut-free and contraction-free labelled sequent calculi for the large family of modal logics whose Kripke semantics are defined by geometric (first-order) formulae. The proof of cut-elimination is general in the sense that it applies uniformly to every modal logic defined by geometric formulae. This result has been extended to labelled sequent calculi for intermediate and other non-classical logics [6] and indeed to arbitrary first-order formulae [7]. See also Viganò [21] where non-classical logics with semantics defined by Horn formulae are investigated using cut-free labelled calculi introduced therein.

We begin by extending in the natural way the usual labelled sequent calculus for $\mathrm{K}$ to a labelled sequent calculus for $\mathrm{Kt}$.

\section{Definition 3 (The labelled sequent calculus G3Kt[17]).}

$$
\begin{gathered}
\overline{\mathcal{R}, x: p, x: \bar{p}, \Gamma}(\text { id) } \\
\frac{\mathcal{R}, x: A, x: B, \Gamma}{\mathcal{R}, x: A \vee B, \Gamma}(\vee) \quad \frac{\mathcal{R}, x: A, \Gamma \quad \mathcal{R}, x: B, \Gamma}{\mathcal{R}, x: A \wedge B, \Gamma}(\wedge) \\
\frac{\mathcal{R}, R y x, y: A, \Gamma}{\mathcal{R}, x: \mathbf{\square} A, \Gamma}(\mathbf{\square})^{*} \frac{\mathcal{R}, R x y, y: A, \Gamma}{\mathcal{R}, x: \square A, \Gamma}(\square)^{*} \\
\frac{\mathcal{R}, R y x, y: A, x: \diamond A, \Gamma}{\mathcal{R}, R y x, x: \diamond A, \Gamma}(\diamond) \quad \frac{\mathcal{R}, R x y, y: A, x: \diamond A, \Gamma}{\mathcal{R}, R x y, x: \diamond A, \Gamma}(\diamond)
\end{gathered}
$$

The $(\square)$ and $(\mathbf{\square})$ rules have a side condition: $(*)$ the variable $y$ does not occur in the conclusion. When a variable is not allowed to occur in the conclusion of an inference, we refer to it as an eigenvariable.

Following the method in [17, the rule $l(h, i, j, k)$ corresponding to the ScottLemmon axiom $\boldsymbol{\square}^{h} \square^{j} \bar{p} \vee \nabla^{i}{ }^{k} p$ is given below. We use the notation $R^{n} x z$ to represent a relational sequence $R x y_{1}, R y_{1} y_{2}, \ldots, R y_{n-1} z$ of length $n$.

$$
\frac{\mathcal{R}, R^{i} v x, R^{k} u x, R^{h} w v, R^{j} w u, v: \Delta, u: \Delta^{\prime}, \Gamma}{\mathcal{R}, R^{h} w v, R^{j} w u, v: \Delta, u: \Delta^{\prime}, \Gamma} l(h, i, j, k)^{*}
$$

(*) All variables occurring in the relational atoms $R^{i} v x, R^{k} u x$ with the exception of $v$ and $u$ are eigenvariables.

Remark 1. In the rule above, some care is needed in the boundary case when some of the parameters $h, i, j$, and $k$ are zero. The table below specifies the instances of the rule depending on whether the parameter is greater than zero (marked with $>$ ), or equal to zero (marked with 0 ): 


\begin{tabular}{|c|c|c|c|c|c|}
\hline $\mathrm{h}$ & $\mathrm{j}$ & $\mathrm{i}$ & $\mathrm{k}$ & Premise & Conclusion \\
\hline$>$ & $>$ & $>$ & $>$ & $\mathcal{R}, R^{i} v x, R^{k} u x, R^{h} w v, R^{j} w u, v: \Delta, u: \Delta^{\prime}, \Gamma$ & $\mathcal{R}, R^{h} w v, R^{j} w u, v: \Delta, u: \Delta^{\prime}, \Gamma$ \\
\hline 0 & $>$ & $>$ & $>$ & $\mathcal{R}, R^{i} v x, R^{k} u x, R^{j} v u, v: \Delta, u: \Delta^{\prime}, \Gamma$ & $\mathcal{R}, R^{j} v u, v: \Delta, u: \Delta^{\prime}, \Gamma$ \\
\hline 0 & $>$ & $>$ & 0 & $\mathcal{R}, R^{i} v u, R^{j} w u, v: \Delta, u: \Delta^{\prime}, \Gamma$ & $\mathcal{R}, R^{j} v u, v: \Delta, u: \Delta^{\prime}, \Gamma$ \\
\hline$>$ & 0 & 0 & $>$ & $\mathcal{R}, R^{k} u v, R^{h} u v, v: \Delta, u: \Delta^{\prime}, \Gamma$ & $\mathcal{R}, R^{h} u v, v: \Delta, u: \Delta^{\prime}, \Gamma$ \\
\hline 0 & 0 & $>$ & $>$ & $\mathcal{R}, R^{i} v x, R^{k} v x, v: \Delta, v: \Delta^{\prime}, \Gamma$ & $\mathcal{R}, v: \Delta, v: \Delta^{\prime}, \Gamma$ \\
\hline 0 & 0 & $>$ & 0 & $\mathcal{R}, R^{i} v v, v: \Delta, v: \Delta^{\prime}, \Gamma$ & $\mathcal{R}, v: \Delta, v: \Delta^{\prime}, \Gamma$ \\
\hline$>$ & $>$ & $>$ & 0 & $\mathcal{R}, R^{i} v u, R^{h} w v, R^{j} w u, v: \Delta, u: \Delta^{\prime}, \Gamma$ & $\mathcal{R}, R^{h} w v, R^{j} w u, v: \Delta, u: \Delta^{\prime}, \Gamma$ \\
\hline 0 & 0 & 0 & 0 & $\mathcal{R}, v: \Delta, u: \Delta^{\prime}, \Gamma$ & $\mathcal{R}, v: \Delta, u: \Delta^{\prime}, \Gamma$ \\
\hline
\end{tabular}

Although there are sixteen cases to consider, we only give eight of these as the others are similar. For some entries in the table, the equality symbol that arises ( $R^{0} u v$ is taken to be $u=v$ ) has been eliminated by suitable argumentation. This argumentation can be formalised using the equality rules specified by Negri [17. In particular, when $i=k=0$ and $h>0, j>0$ the rule obtained in this way has the following form.

$$
\frac{\mathcal{R}, R^{h} w v, R^{j} w v, v: \Delta, v: \Delta^{\prime}, \Gamma}{\mathcal{R}, R^{h} w v, R^{j} w u, v: \Delta, u: \Delta^{\prime}, \Gamma} l(h, i, j, k)^{*}
$$

Negri [17 does not explicitly consider structural rules of this form (observe how $v: \Delta^{\prime}$ in the premise becomes $u: \Delta^{\prime}$ in the conclusion). The results in this paper apply to such rules as well, by extending Negri's arguments in order to justify the elimination of the equality symbol.

The following contraction and weakening rules are admissible 17 in G3Kt + $l(h, i, j, k)$.

$$
\frac{\mathcal{R}, \mathcal{Q}, \mathcal{Q}, \Delta, \Delta, \Gamma}{\mathcal{R}, \mathcal{Q}, \Delta, \Gamma}(\mathrm{ctr}) \quad \frac{\mathcal{R}, \Gamma}{\mathcal{R}, \mathcal{Q}, \Gamma, \Delta}(\mathrm{wk})
$$

Theorem 2 ([17]). Let $S$ be any finite set of Scott-Lemmon axioms. $A \in K t+S$ iff $x: A$ is derivable in $S K T+S^{\prime}$, where $S^{\prime}=\left\{l(h, i, j, k) \mid \mathbf{\square}^{h} \square^{j} \bar{p} \vee{ }^{i}{ }^{k} p \in S\right\}$.

\section{Interpreting a display sequent as a labelled UT}

In this section we show how to translate (back and forth) a display sequent into a labelled directed graph whose underlying undirected graph is a tree.

We write $V=V_{1} \sqcup V_{2}$ to mean that $V=V_{1} \cup V_{2}$ and $V_{1} \cap V_{2}=\emptyset$. The multiset union of multisets $M_{1}$ and $M_{2}$ is denoted $M_{1} \uplus M_{2}$. A labelling function $L$ is a map from a set $V$ to a multiset of tense formulae. For labelling functions $L_{1}$ and $L_{2}$ on the set $V_{1}$ and $V_{2}$ respectively, let $L_{1} \cup L_{2}$ be the labelling function on $V_{1} \cup V_{2}$ defined as follows:

$$
L_{1} \cup L_{2}(x)= \begin{cases}L_{1}(x) & x \in V_{1}, x \notin V_{2} \\ L_{2}(x) & x \notin V_{1}, x \in V_{2} \\ L_{1}(x) \uplus L_{2}(x) & x \in V_{1}, x \in V_{2}\end{cases}
$$


A labelled graph $(V, E, L)$ is a directed graph $(V, E)(V \neq \emptyset)$ equipped with a labelling function $L$ on $V$.

Definition 4 (Labelled graph isomorphism). We say that two labelled graphs $u_{1}=\left(V_{1}, E_{1}, L_{1}\right)$ and $u_{2}=\left(V_{2}, E_{2}, L_{2}\right)$ are isomorphic (written $u_{1} \cong u_{2}$ ) if and only if there is an isomorphism $f: V_{1} \rightarrow V_{2}$ such that:

(i) for every $x, y \in V_{1},(x, y) \in E_{1}$ iff $(f x, f y) \in E_{1}$

(ii) for every $x \in V, L(x)=L(f x)$.

Definition 5 (Labelled UT). A labelled graph whose underlying (undirected) graph is a tree is termed a UT (underlying tree).

Example 1. Assuming that the nodes are decorated with multisets of formulae, the following two graphs represent labelled UTs:
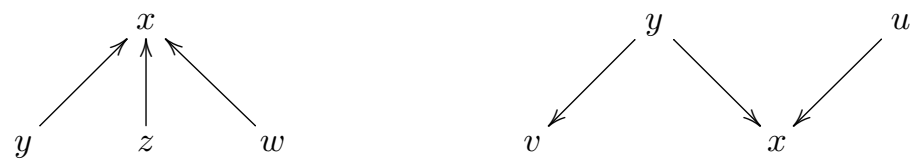

Interpreting a display sequent $\boldsymbol{\Gamma}$ as a labelled UT. Every display sequent has a natural interpretation as a labelled tree with two types of directed edges: $\stackrel{\circ}{\rightarrow}$ and $\stackrel{\bullet}{\rightarrow}$. If we interpret every directed edge $\alpha \stackrel{\bullet}{\rightarrow} \beta$ as the directed edge $\beta \stackrel{\circ}{\leftarrow} \alpha$, we can then interpret every display sequent as a connected labelled graph with a single type of directed edge (so we can drop the o symbol altogether). Moreover, it is easy to see that its underlying graph (i.e. the undirected graph obtained obtained treating all edges as undirected) has no cycles.

Remark 2. Every display sequent $\Gamma$ can be interpreted naturally as a UT.

Example 2. First interpret the display sequent $A, \circ\{B, \bullet\{\}\}, \bullet\{D, E, \bullet\{F\}, \circ\{G\}\}$ as the labelled tree with two types of directed edges, below left. Next, convert this labelled tree to a labelled graph (with a single type of directed edge) by reading each $\alpha \stackrel{\bullet}{\rightarrow}$ as $\alpha \leftarrow \beta$ (below right).

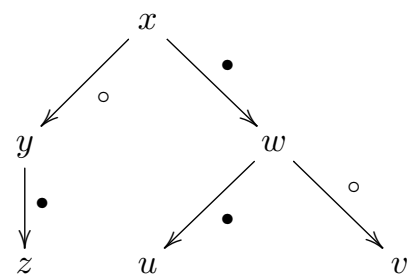

$L(x)=\{A\}$
$L(w)=\{D, E\}$
$L(y)=\{B\}$

$L(u)=\{F\}$

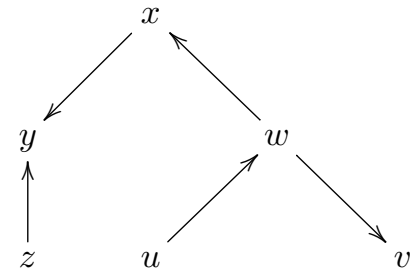

$L(z)=\emptyset$

$L(v)=\{G\}$ 
For concreteness let us formally define the map $d u$ from a display sequent to a UT. Let $\mathbb{N}<\mathbb{N}$ denote the set of finite sequences on $\mathbb{N}$.

Given $(x) \in \mathbb{N}^{<\mathbb{N}}$ and a display sequent $\Gamma$, consider the following recursive definition for $d u_{(x)}(\Gamma)$ on the depth of $\Gamma$ :

1. Base case. $\Gamma=A_{1}, \ldots, A_{M}$. A pictorial representation is given below right.

$$
d u_{(x)}\left(A_{1}, \ldots, A_{M}\right)=\left(\{(x)\}, \emptyset, x \mapsto\left\{A_{1}, \ldots, A_{n}\right\}\right) \quad A_{1}, \ldots, A_{M}
$$

2. Inductive case. $\Gamma=A_{1}, \ldots, A_{M}, \oslash_{1}\left\{X_{1}\right\}, \ldots, \varnothing_{N}\left\{X_{N}\right\}$ where $\varnothing_{j} \in\{\circ, \bullet\}$.

Since each $\bigcirc_{j}\left\{X_{j}\right\}$ has strictly smaller depth than $\Gamma$, the following are welldefined:

$$
d u_{(x j)}\left(\bigcirc_{j}\left\{X_{j}\right\}\right)=\left(V_{j}, E_{j}, L_{j}\right) \text { for } 1 \leq j \leq N
$$

Define $d u_{(x)}(\Gamma)=(V, E, L)$ such that

$$
\begin{aligned}
& V=\{(x)\} \cup V_{1} \cup \ldots \cup V_{N} \\
& E=\left\{((x),(x j)) \mid \bigcirc_{j}=\circ\right\} \cup\left\{((x j),(x)) \mid \bigcirc_{j}=\bullet\right\} \\
& L=L_{(x)} \cup L_{1} \cup \ldots \cup L_{N}
\end{aligned}
$$

A pictorial representation is given below. The orientation of the arrows is determined by $\nabla_{j}$. If ${{ }_{j}}_{j}=0$ then the arrow directs away from $(x)$; if $\nabla_{j}=\bullet$ then the arrow directs towards $(x)$

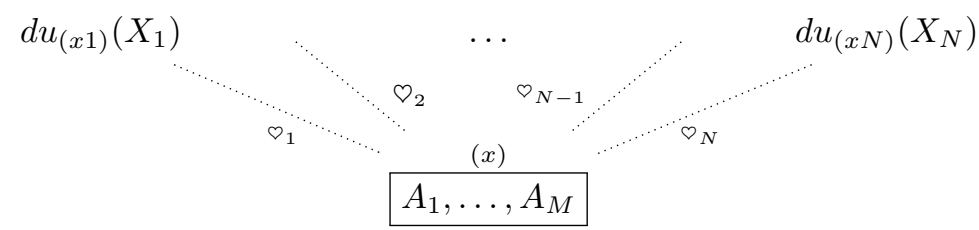

Remark 3. Every comma occurring in the display sequent $\Gamma$ is associated (though not necessarily a one-to-one association) with a vertex in $d u(\Gamma)$.

Example 3. Given $\Gamma=A, \circ\{B, \bullet\{C\}\}, \bullet\{D\}$, the UT $d u_{(1)}(\Gamma)=(V, E, L)$ is computed below:

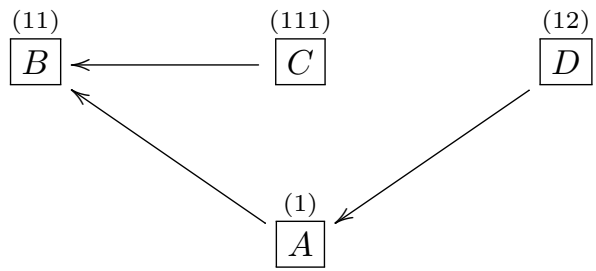

Note that in practice we use the more familiar symbols $x, y, z, \ldots$ to denote labels. The numerical labels are used here for technical convenience. 
Definition $6(u[v]$ notation). We write $u[v]$ to mean the labelled graph containing labelled subgraphs $u[]$ and $v$ which have a single vertex $x$ in common such that the label of $x$ in $u[v]$ is the union of $L(x)$ from $u[]$ and $v$.

Example 4. Consider the labelled graph $u[v]-x$ is the common vertex between $u[]$ and $v$-shown below right. Then either of the two labelled graphs below right may be $v$, and the other will be $u[]$.
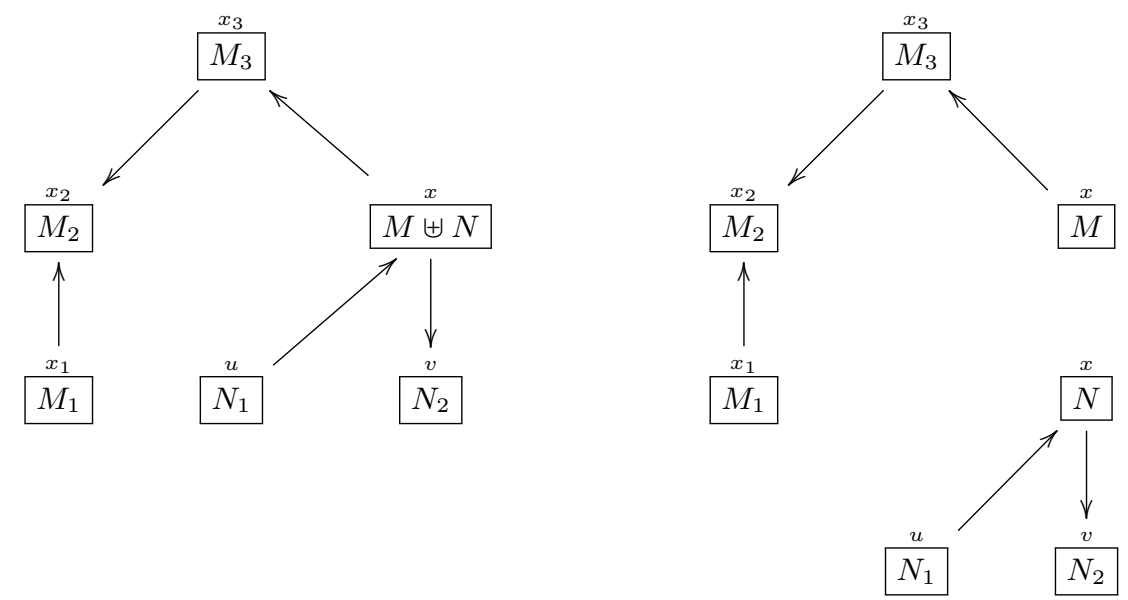

If $u[v]=(V, E, L)$, then there exist partitions $V=V_{1} \sqcup\{x\} \sqcup V_{2}, E=E_{1} \sqcup E_{2}$, and $L_{1}$ and $L_{2}$ such that $L=L_{1} \cup L_{2}$, where $u[]=\left(V_{1} \sqcup\{x\}, E_{1}, L_{1}\right)$ and $v=$ $\left(V_{2} \sqcup\{x\}, E_{2}, L_{2}\right)$. In particular, $L(x)=L_{1}(x) \uplus L_{2}(x)$. Note that when $u[v]$ is a labelled UT, then $u[]$ and $v$ must necessarily be labelled UTs.

We have seen that every display sequent defines (up to isomorphism) a labelled UT. With a slight abuse of notation, we will use the display sequent notation to denote a labelled UT. For example, we will write $u[X]$ to mean the labelled graph such that the labelled graph $u[]$ and the labelled UT $d u(X)$ are subgraphs with a single common vertex. The context will make it clear if we are referring to a display sequent or a labelled UT.

The translation from a display sequent to a labelled UT extends naturally to a translation from a display sequent rule to a labelled UT rule. This leads us to the definition of the following calculus.

Definition 7 (UT calculus). Every sequent in this calculus is a labelled UT.

$$
\begin{aligned}
& \overline{u[p, \bar{p}]}(\text { id })_{u} \quad \frac{u[A] \quad u[B]}{u[A \wedge B]}(\wedge)_{u} \quad \frac{u[A, B]}{u[A \vee B]}(\vee)_{u} \\
& \frac{A, \circ\{X\}}{\mathbf{\square} A, X}(\mathbf{\square})_{u} \frac{u[\circ\{\Delta, A\}, \diamond A]}{u[\circ\{\Delta\}, \diamond A]}(\diamond)_{u} \frac{u[\circ\{\Delta, \diamond A\}, A]}{u[\circ\{\Delta, \diamond A\}]}(\diamond)_{u} \\
& \frac{u[\circ\{A\}]}{u[\square A]}(\square)_{u} \quad \frac{u[\Gamma]}{u[\Gamma, \Delta]}(w k)_{u} \quad \frac{u[\Delta, \Delta]}{u[\Delta]}(c t r)_{u}
\end{aligned}
$$


For convenience, we drop the subscript $(x)$ and write $d u$ for $d u_{(x)}$.

Recall that SKT $+d(h, i, j, k)$ (see below left) is a calculus for the extension of Kt with the Scott-Lemmon axiom $\square^{h} \square^{j} \bar{p} \vee \diamond^{i}{ }^{k} p$. We define the UT rule $u(h, i, j, k)$ as below right.

$$
\frac{\Gamma, \circ^{i}\left\{\bullet^{k}\{\Delta\}\right\}}{\Gamma, \bullet^{h}\left\{\mathrm{o}^{j}\{\Delta\}\right\}} d(h, i, j, k) \quad \frac{u\left[\circ^{i}\left\{\bullet^{k}\{\Delta\}\right\}\right]}{u\left[\bullet^{h}\left\{\mathrm{o}^{j}\{\Delta\}\right\}\right]} u(h, i, j, k)
$$

Since display sequents may be interpreted as trees with two types of edges (o-edges and •-edges), they possess a root node, whereas UTs do not possess a root in general. Nevertheless, the underlying tree structure of a UT permits us to view any node as the root, and the lemma below ensures that we obtain deductively equivalent labelled UTs via the residuation rules regardless of the node where we begin the translation.

Lemma 1. For every $\Gamma$ and $\Delta: d u(\Gamma, \circ\{\Delta\}) \cong d u(\bullet\{\Gamma\}, \Delta)$

Proof. Let $(V, E, L)=d u(\Gamma, \circ\{\Delta\})$. Then there exists $x, y \in V$ and $(x, y) \in E$ such that $V=V_{1} \sqcup\{x\} \sqcup V_{2} \sqcup\{y\}$ and $E=E_{1} \sqcup E_{2} \sqcup\{(x, y)\}$ and $d u(\Gamma)=$ $\left(V_{1} \sqcup\{x\}, E_{1},\left.L\right|_{V_{1} \sqcup\{x\}}\right)$ and $d u(\Delta)=\left(V_{2} \sqcup\{y\}, E_{2},\left.L\right|_{V_{2} \sqcup\{y\}}\right)$. In particular, observe that that comma displayed in $\Gamma, \circ\{\Delta\}$ corresponds to $x$ and the nesting where $\Delta$ occurs corresponds to $y$.

Now consider $d u(\bullet\{\Gamma\}, \Delta)=\left(V^{\prime}, E^{\prime}, L^{\prime}\right)$. There exists $u, v \in V^{\prime}$ and $(u, v) \in$ $E$ such that $V^{\prime}=V_{1}^{\prime} \sqcup\{u\} \sqcup V_{2}^{\prime} \sqcup\{y\}$ and $E^{\prime}=E_{1}^{\prime} \sqcup E_{2}^{\prime} \sqcup\{(u, v)\}$ and $d u(\Gamma)=$ $\left(V_{1}^{\prime} \sqcup\{u\}, E_{1}^{\prime},\left.L^{\prime}\right|_{V_{1}^{\prime} \sqcup\{u\}}\right)$ and $d u(\Delta)=\left(V_{2}^{\prime} \sqcup\{v\}, E_{2}^{\prime},\left.L^{\prime}\right|_{V_{2}^{\prime} \sqcup\{v\}}\right)$. In particular, observe that that comma displayed in $\bullet\{\Gamma\}, \Delta$ corresponds to $v$ and the nesting where $\Gamma$ occurs corresponds to $u$.

It follows that there are isomorphisms witnessing each of the following such that $x$ maps to $u$ (first line) and $y$ maps to $v$ (second line).

$$
\begin{aligned}
& d u(\Gamma)=\left(V_{1} \sqcup\{x\}, E_{1},\left.L\right|_{V_{1} \sqcup\{x\}}\right) \cong\left(V_{1}^{\prime} \sqcup\{u\}, E_{1}^{\prime},\left.L^{\prime}\right|_{V_{1}^{\prime} \sqcup\{u\}}\right)=d u(\Gamma) \\
& d u(\Delta)=\left(V_{2} \sqcup\{y\}, E_{2},\left.L\right|_{V_{2} \sqcup\{y\}}\right) \cong\left(V_{2}^{\prime} \sqcup\{v\}, E_{2}^{\prime},\left.L^{\prime}\right|_{V_{2}^{\prime} \sqcup\{v\}}\right)=d u(\Delta)
\end{aligned}
$$

Taking the graph union of these disjoint graphs:

$$
\begin{aligned}
& \left(V_{1} \sqcup\{x\} \sqcup V_{2} \sqcup\{y\}, E_{1} \sqcup E_{2},\left.\left.L\right|_{V_{1} \sqcup\{x\}} \cup L\right|_{V_{2} \sqcup\{y\}}\right) \cong \\
& \left(V_{1}^{\prime} \sqcup\{u\} \sqcup V_{2}^{\prime} \sqcup\{y\}, E_{1}^{\prime} \sqcup E_{2}^{\prime},\left.\left.L^{\prime}\right|_{V_{1}^{\prime} \sqcup\{u\}} \cup L^{\prime}\right|_{V_{2}^{\prime} \sqcup\{v\}}\right)
\end{aligned}
$$

Adding the edge $(x, y)$ on the left and $(u, v)$ on the right, we get $(V, E, L) \cong$ $\left(V^{\prime}, E^{\prime}, L^{\prime}\right)$.

Interpreting a labelled UT as a display sequent. Given a UT $u=\langle V, E, L\rangle$ we first pick a vertex $x \in V$ to compute the display sequent $u d_{x}(u)$. If $E=\emptyset$, then $u d(u)=L(x)$ is the desired display sequent. Otherwise, for all $n$ forward looking edges $\left(x, y_{i}\right) \in E$ (with $1 \leq i \leq n$ ) where $y_{i}$ is the common label of $u=u\left[v_{i}\right]$ and $v_{i}$, and for all $k$ backward looking edges $\left(z_{j}, x\right) \in E$ (with $1 \leq j \leq k$ ) where 
$z_{j}$ is the common label of $u=u\left[w_{j}\right]$ and $w_{j}$, we define the image of $u d_{x}(u)$ as the display sequent

$$
L(x), \circ\left\{u d_{y_{1}}\left(v_{1}\right)\right\}, \ldots, \circ\left\{u d_{y_{n}}\left(v_{n}\right)\right\}, \bullet\left\{u d_{z_{1}}\left(w_{1}\right)\right\}, \ldots, \bullet\left\{u d_{y_{k}}\left(v_{k}\right)\right\}
$$

Since the UTs $v_{1}, \ldots, v_{n}, w_{1}, \ldots, w_{k}$ are smaller than $u$, the recursive definition of $u d$ is well-founded.

Lemma 2. For any $U T u=\langle V, E, L\rangle$, and for any vertices $x, y \in V$, the display sequent $u d_{x}(u)$ is derivable from $u d_{y}(u)$ via the residuation rules $(r f)$ and $(r p)$.

Proof. Follows by Lemma 1 .

When translating a labelled UT we must choose a vertex as the starting point of our translation. This lemma states that all display sequents obtained from choosing a different vertex are mutually derivable from one another. In fact, all such display sequents are display equivalent, meaning they are derivable from each other by use of the residuation rules (rp) and (rf) only. To clarify the translation procedure, we provide an example below of the various display sequents obtained from translating at a different vertex initially.

Example 5. Suppose we are given the labelled UT $u=\langle V, E, L\rangle$ where $V=$ $\{x, y, z\}, E=\{(x, y),(z, x)\}, L(x)=\{A\}, L(y)=\{B, C\}$, and $L(z)=\{D\}$. A pictorial representation of the labelled UT $u$ is given on the left with the corresponding display sequent translations on the right:

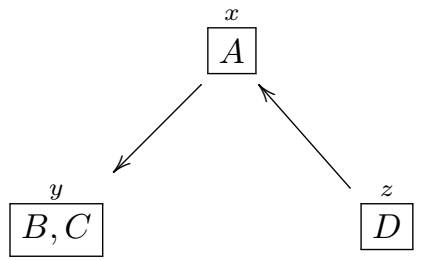

$$
\begin{aligned}
& u d_{x}(u)=A, \circ\{B, C\}, \bullet\{D\} \\
& u d_{y}(u)=B, C, \bullet\{A, \bullet\{D\}\} \\
& u d_{z}(u)=D, \circ\{A, \circ\{B, C\}\}
\end{aligned}
$$

When providing the construction of an effective translation between display calculus proofs and UT calculus proofs, we make use of the notation $\Gamma, \Delta$ for display sequents and $u[v]$ for corresponding labelled UTs (under the translation). The following lemma ensures that the pieces of the display sequent $\Gamma$ and $\Delta$, and the pieces of the labelled UT $u[]$ and $v$, correctly map to each other under our translation functions.

Lemma 3. (i) For every $\Gamma$ and $\Delta, d u(\Gamma, \Delta)$ is the $U T u[v]$, where $v$ is the $U T d u(\Delta)$ and $u[]$ is the $U T d u(\Gamma)$.

(ii) For every $U T u[v], u d(u[v])$ is the display sequent $\Gamma, \Delta$ (up to display equivalence) where $\Gamma=u d(u[])$ and $\Delta=u d(v)$.

Proof. By construction of $d u$ and $u d$. 
Theorem 3 (Translating derivations: SKT $+S$ and UT calculus $\left.+S^{\prime}\right)$. Let $S$ be any finite set of $d(h, i, j, k)$ rules and $S^{\prime}$ be the set $\{u(h, i, j, k) \mid d(h, i, j, k) \in$ S\}. Then:

(i) Let $\delta$ be a derivation of $\Gamma$ in $S K T+S$. Then there is an effective translation of $\delta$ to a derivation $\delta^{\prime}$ of $d u(\Gamma)$ in the UT calculus with $S^{\prime}$.

(ii) Let $\delta$ be a derivation of the labelled UT $u$ in the UT calculus with $S^{\prime}$. Then there is an effective translation of $\delta$ to a derivation of $u d(g)$ in $S K T+S$.

Proof. (i) Induction on the height of $\delta$.

Base case. $d u(\Gamma, p, \bar{p})$ is a UT of the form $u[p, \bar{p}]$ (Lemma $3(\mathrm{i})$ ) and is hence an initial sequent in the UT calculus.

Inductive case. It suffices to simulate each rule instance of SKT in the UT calculus. Every rule in SKT other than (rf), (rp), ( $)$ and $(\bullet)$ has the form below left for suitable $Y_{1}$ and $Y_{0}$; moreover, there is a corresponding rule in the UT calculus as shown below right.

$$
\frac{\Gamma, Y_{1}}{\Gamma, Y_{0}}(\mathbf{r}) \quad \frac{u\left[\Gamma, Y_{1}\right]}{u\left[\Gamma, Y_{0}\right]}(\mathbf{r})_{u}
$$

The induction hypothesis gives us a derivation of $d u\left(\Gamma, Y_{1}\right)=u\left[\Gamma, Y_{1}\right]$. Applying $(\mathrm{r})_{u}$ we get $u\left[\Gamma, Y_{0}\right]=d u\left(\Gamma, Y_{0}\right)$ as required.

We consider the remaining rules below.

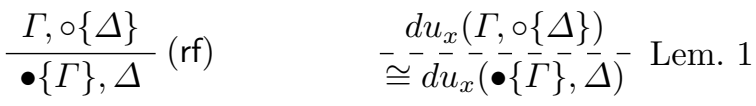

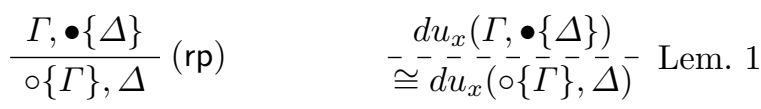

$$
\begin{aligned}
& \frac{\Gamma, \bullet\{A\}}{\Gamma, \boldsymbol{\square} A}(\mathbf{\square}) \quad \begin{array}{c}
d u_{x}(\Gamma, \bullet\{A\}) \\
\frac{\circ\{\bar{\Gamma}\}, A}{\Gamma, \boldsymbol{\square} A}(\mathbf{\square})
\end{array}
\end{aligned}
$$

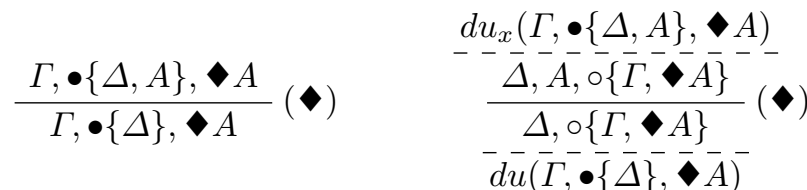

(iii) Induction on the height of $\delta$. The argument is similar to the above case and uses Lemma 3 (ii).

\section{From labelled UTs to labelled sequents}

We identify a subclass of labelled sequents which we call G3Kt(UT) sequents, and prove that they correspond to labelled UT graphs. Due to the relations of the latter with the display calculi shown in the previous section, it follows that every derivation in the $\mathrm{SKT}+u(h, i, j, k)$ calculus corresponds to a derivation in the labelled calculus restricted to $\mathrm{G} 3 \mathrm{Kt}(U T)$ sequents. 
Transforming a labelled UT $u=(V, E, L)$ into a labelled sequent $\mathcal{R}, \Gamma$. Define $\mathcal{R}=\{R x y \mid(x, y) \in E\}$ and

$$
\Gamma=\biguplus_{x \in V, L(x) \neq \emptyset} x: L(x)
$$

where $x: L(x)$ represents the multiset $L(x)$ with each formula prepended with a label $x$.

Example 6. The UT $u=\langle V, E, L\rangle$ where $V=\{x, y, z\}, E=\{(x, y),(z, x)\}$, $L(x)=\{A\}, L(y)=\{B\}$, and $L(z)=\{C\}$ corresponds to the labelled sequent $R x y, R z x, x: A, y: B, z: C$.

Transforming a labelled sequent $\mathcal{R}, \Gamma$ into a labelled graph $(V, E, L)$. Let $V$ be the set of all labels occurring in $\mathcal{R}, \Gamma$. Define

$$
E=\{(x, y) \mid R x y \in \mathcal{R}\} \quad L(x)=\{\text { multiset of formulae with label } x \text { in } \Gamma\}
$$

Example 7. The labelled sequent Rxy, Ryz, Rux, $x: A, z: B, z: C, u: D$ becomes the UT $u=\langle V, E, L\rangle$ where $V=\{x, y, z, u\}, E=\{(x, y),(y, z),(u, x)\}$, $L(x)=\{A\}, L(y)=\emptyset, L(z)=\{B, C\}$ and $L(u)=\{D\}$.

The reader will observe that the translations are obtained rather directly. This is because the main difference between a labelled graph and a labelled sequent is notation. The main step of the translation was already established in the previous section. Our interest in this work is the image of a display sequent in the labelled calculus. This motivates the following definitions.

Definition 8 (G3Kt $(U T)$ sequent). A labelled sequent whose image (under the above translation) is a labelled $U T$ is called a $\mathrm{G} 3 \mathrm{Kt}(U T)$ sequent.

Definition 9 (G3Kt $(U T)$ calculus). Define the calculus G3Kt(UT) to be the labelled calculus restricted to G3Kt(UT) sequents and with weakening and contraction defined as follows:

$$
\frac{\mathcal{R}, \Gamma}{\mathcal{R}, \mathcal{Q}, \Delta, \Gamma}(w k)_{u l}^{*} \quad \frac{\mathcal{R}, \mathcal{Q}, \hat{\mathcal{Q}}, \Delta, \hat{\Delta}, \Gamma}{\mathcal{R}, \mathcal{Q}, \Delta, \Gamma}(c t r)_{u l}^{*}
$$

Weakening has the side condition that the conclusion must be a G3Kt(UT)sequent. Contraction possesses side conditions that ensure it behaves just as the $(c t r)_{u}$ rule:

1. The labelled graph of $\hat{\mathcal{Q}}, \hat{\Delta}$ must be isomorphic to the labelled graph of $\mathcal{Q}, \Delta$.

2. The conclusion must be a G3Kt(UT)-sequent.

3. Both $\mathcal{Q}, \Delta$ and $\hat{\mathcal{Q}}, \hat{\Delta}$ form labelled UTs that share a root, and all other variables in $\hat{\mathcal{Q}}, \hat{\Delta}$ do not appear in the conclusion of the inference, i.e. they are eigenvariables. 
We use the notation $(\mathrm{r})_{\text {ul }}$ to indicate the remaining inference rules of G3Kt(UT).

For $h, i, j, k \in \mathbb{N}$, define $u l(h, i, j, k)$ as follows:

$$
\frac{\mathcal{R}, R^{i} v x, R^{k} u x, v: \Delta, u: \Delta^{\prime}, \Gamma}{\mathcal{R}, R^{h} w v, R^{j} w u, v: \Delta, u: \Delta^{\prime}, \Gamma} u l(h, i, j, k)^{*}
$$

The asterisk indicates the following side conditions: (i) all variables occurring in $R^{i} v x, R^{k} u x$ with the exception of $v$ and $u$ are eigenvariables and (ii) all variables occurring in $R^{h} w v, R^{j} w u$ with the exception of $v$ and $u$ are fresh.

Remark 4. Similar to the presentation of the $l(h, i, j, k)$ rules (cf. Remark 1), we provide the table below showing the different instances of the rule depending on the values of the parameters $h, i, j$, and $k$. The reduction in cases is due to the fact that we allow the $u l(h, i, j, k)$ rules to relabel formulae from premise to conclusion-an action which is not allowed for the $l(h, i, j, k)$ rules.

\begin{tabular}{|c|c|c|}
\hline $\mathrm{i}$ & $\mathrm{k}$ & Premise \\
\hline$>$ & $>$ & $\mathcal{R}, R^{i} v x, R^{k} u x, v: \Delta, u: \Delta^{\prime}, \Gamma$ \\
\hline 0 & $>$ & $\mathcal{R}, R^{k} u v, v: \Delta, u: \Delta^{\prime}, \Gamma$ \\
\hline$>$ & 0 & $\mathcal{R}, R^{i} v u, v: \Delta, u: \Delta^{\prime}, \Gamma$ \\
\hline 0 & 0 & $\mathcal{R}, v: \Delta, v: \Delta^{\prime}, \Gamma$ \\
\hline
\end{tabular}

\begin{tabular}{|c|c|c|}
\hline $\mathrm{h}$ & $\mathrm{j}$ & Conclusion \\
\hline$>$ & $>$ & $\mathcal{R}, R^{h} w v, R^{j} w u, v: \Delta, u: \Delta^{\prime}, \Gamma$ \\
\hline 0 & $>$ & $\mathcal{R}, R^{j} w u, w: \Delta, u: \Delta^{\prime}, \Gamma$ \\
\hline$>$ & 0 & $\mathcal{R}, R^{h} w v, v: \Delta, w: \Delta^{\prime}, \Gamma$ \\
\hline 0 & 0 & $\mathcal{R}, w: \Delta, w: \Delta^{\prime}, \Gamma$ \\
\hline
\end{tabular}

To see that the $\mathrm{G} 3 \mathrm{Kt}(U T)+u l(h, i, j, k)$ calculus is well-defined, it suffices to observe that the conclusion of every G3Kt rule is a $\mathrm{G} 3 \mathrm{Kt}(U T)$ sequent given that the premise(s) is (are) G3Kt $(U T)$ sequents.

Lemma 4. If the premise of a G3Kt $(U T)+u l(h, i, j, k)$ inference is a G3Kt $(U T)$ sequent, then the conclusion is an G3Kt(UT)-sequent.

Proof. We argue the result for the $(\mathrm{wk})_{u l},(\mathrm{ctr})_{u l},(\mathbf{\square})_{u l}$, and $u l(h, i, j, k)$ rules since all other cases are similar or trivial.

Case 1 and 2. These cases follow from the side conditions on the $(w k)_{u l}$ and $(\mathrm{ctr})_{u l}$ rules, which only allow application of the rule when the result is a $\mathrm{G} 3 \mathrm{Kt}(U T)$ sequent.

Case 3. Assume that $\mathcal{R}, R y x, y: A, \Gamma$ is a $\operatorname{GKKt}(U T)$-sequent and that $u=$ $\langle V, E, L\rangle$ is the corresponding UT. Since $y$ is an eigenvariable, the conclusion $\mathcal{R}, x: \square, \Gamma$ gives a labelled graph $u^{\prime}=\left\langle V^{\prime}, E^{\prime}, L^{\prime}\right\rangle$ where $V^{\prime}=V-\{y\}$, $E^{\prime}=E-\{(y, x)\}, L^{\prime}(y)$ is undefined, $L^{\prime}(x)$ is equal to $L(x)$ extended with $x \mapsto\left\{\mathbb{\square} A\right.$, and $L^{\prime}$ is equal to $L$ for all other labels in $V^{\prime}$.

Case 4 . We prove the claim for when $h, i, j, k>0$ since other cases are similar. Assume that the premise $\mathcal{R}, R^{i} x y, R^{k} z y, \Gamma$ is a $\mathrm{G} 3 \mathrm{Kt}(U T)$-sequent with all variables $y_{m}$ strictly between $x$ and $z$ eigenvariables. Observe that in $u=$ $\langle V, E, L\rangle$ there is a path of length $i+k$ from the node $x$ to $z$ where the first $i$ edges are forward looking, and the last $k$ edges are backwards looking. Observe that the UT $u^{\prime}=\left\langle V^{\prime}, E^{\prime}, L^{\prime}\right\rangle$ of the conclusion $\mathcal{R}, R^{h} w x, R^{j} w z, \Gamma$ will contain a path of length $h+j$ from the node $x$ to $z$ where the first $h$ edges are backwards 
looking, and the last $j$ edges are forwards looking. Due to the eigenvariable condition on all nodes $y_{m}$ strictly between $x$ and $z$, it cannot be the case that an edge given by $\mathcal{R}$ contains a label $y_{m}$, and it must be the case that $L\left(y_{m}\right)=\emptyset$ (thus ensuring $u^{\prime}$ is connected). Also, all new nodes along the $h+j$-path strictly between $x$ and $z$ will be fresh (thus ensuring $u^{\prime}$ is free of cycles). Hence, $u^{\prime}$ will be a UT.

Lemma 5 (Translating derivations: G3Kt $(U T)+S$ and $\mathbf{U T}$ calculus $+S^{\prime}$ ). Let $S$ be any finite set of $u l(h, i, j, k)$ rules and $S^{\prime}=\{u(h, i, j, k) \mid u l(h, i, j, k) \in$ $S\}$. Then

(i) Let $\delta$ be a derivation of $x: A$ in $G 3 K t(U T)+S$. Then there is an effective translation of $\delta$ to a derivation $\delta^{\prime}$ of $A$ in the UT calculus $+S^{\prime}$.

(ii) Let $\delta$ be a derivation of $A$ in the UT calculus+ $S^{\prime}$. Then there is an effective translation of $\delta$ to a derivation $\delta^{\prime}$ of $x: A$ in $G 3 K t(U T)+S$.

Proof. Follows from the observation that the translation of every rule instance in $\mathrm{G} 3 \mathrm{Kt}(U T)+S$ is a rule instance in the $\mathrm{UT}$ calculus $+S^{\prime}$ and vice versa.

Combining the previous results we obtain:

Theorem 4 (Translating derivations: SKT $+S$ and G3Kt $\left.(U T)+S^{\prime}\right)$. Let $S$ be any finite set of $d(h, i, j, k)$ rules and $S^{\prime}=\{u l(h, i, j, k) \mid d(h, i, j, k) \in S\}$. Then

1. Let $\delta$ be a derivation of $A$ in $S K T+S$. Then there is an effective translation of $\delta$ to a derivation $\delta^{\prime}$ of $x: A$ in $G 3 K t(U T)+S^{\prime}$.

2. Let $\delta$ be a derivation of $x: A$ in $G 3 K t(U T)+S^{\prime}$. Then there is an effective translation $\delta$ to a derivation $\delta^{\prime}$ of $A$ in $S K T+S$.

Proof. Immediate from Theorem 3 and Lemma 4.

Example 8. Below we translate a derivation of $\square \bar{p} \vee \diamond \checkmark p$ in SKT $+d(1,1,1,1)$ to a derivation in $\mathrm{G} 3 \mathrm{Kt}(U T)+u l(1,1,1,1)$.

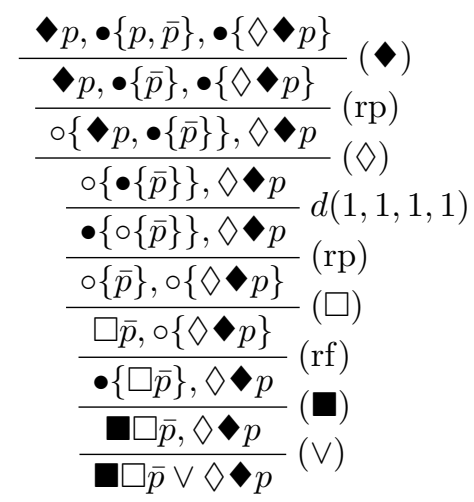

$$
\begin{aligned}
& \frac{R z u, R x u, u: \diamond p, z: p, z: \bar{p}, x: \diamond \diamond p}{R z u, R x u, u: \diamond p, z: \bar{p}, x: \diamond \diamond p}(\diamond)_{u l} \\
& \frac{R z u, R x u, z: \bar{p}, x: \diamond \diamond p}{R y z, R y x, z: \bar{p}, x: \diamond \downarrow p} u l(1,1,1,1) \\
& R y x, y: \square \bar{p}, x: \diamond{ }^{\prime} p \\
& \frac{x: \square \bar{p}, x: \diamond \diamond p}{x: \square \bar{p} \vee \diamond \diamond p}(\vee)_{u l}
\end{aligned}
$$




\section{$5 \quad$ Labelled UTs vs labelled sequents}

In the previous sections, we observed how to embed the display calculus SKT + $S$ (for a finite set $S$ of $d(h, i, j, k)$ rules) in the labelled calculus formalism, in particular, as a proper fragment, which we called G3Kt $(U T)+S^{\prime}\left(S^{\prime}=\right.$ $\{u l(h, i, j, k) \mid d(h, i, j, k) \in S\})$. Indeed, an $\mathrm{G} 3 \mathrm{Kt}(U T)$-sequent is a severe restriction of a labelled sequent since the underlying graph in the former is restricted to a tree. For example, Rxy, Ryx, $x: A, z: B$ is a labelled sequent in the traditional sense, but fails to be a $\mathrm{G} 3 \mathrm{Kt}(U T)$-sequent since it contains the relational cycle $R x y, R y x$ and is not connected due to $z: B$. As a result we have two distinct labelled calculi for Scott-Lemmon extensions of Kt. In this section we investigate the natural question that arises: what is the relationship between these calculi? As seen below, the labelled calculus simulates $\mathrm{G} 3 \mathrm{Kt}(U T)+S^{\prime}$, despite the slightly different rules (i.e. $u l(h, i, j, k)$ ) used by the latter to capture the Scott-Lemmon axioms. The next question is therefore whether the converse also holds, that is, whether the two calculi can represent the same proofs. In the case of the normal minimal tense logic Kt the answer is affirmative.

From G3Kt $(U T)+u l(h, i, j, k)$ to G3Kt $+\boldsymbol{l}(\boldsymbol{h}, \boldsymbol{i}, \boldsymbol{j}, \boldsymbol{k})$. As observed in Remark 11 the structural rules corresponding to $i=k=0$ and $h>0, j>0$ do not match the form of the rules given in [17] (and hence the results in [17] need to be suitably extended to apply to this case). Although an effective translation from $\mathrm{G} 3 \mathrm{Kt}(U T)+u l(h, i, j, k)$ to $\mathrm{G} 3 \mathrm{Kt}+l(h, i, j, k)$ can be defined for this case, in the following we restrict ourselves to $i>0$ or $k>0$.

Lemma 6. ([17], Section 4) The calculus G3Kt $+l(h, i, j, k)$ admits height preserving substitution of variables.

In the following, the requirement that $i>0$ or $k>0$ may be dropped by a slight extension of Negri's arguments (see after Remark 1).

Theorem 5. Let $\delta$ be a derivation of $x: A$ in $\operatorname{GKKt}(U T)+u l(h, i, j, k)$, with $i>0$ or $k>0$. Then there is an effective translation of $\delta$ to a derivation $\delta^{\prime}$ of $x: A$ in $G 3 K t+l(h, i, j, k)$.

Proof. We prove the result by induction on the height of the derivation $\delta$.

Base case. It is easy to see that initial sequents of $\mathrm{G} 3 \mathrm{Kt}(U T)$ are initial sequents of G3Kt.

Inductive step. We show the inductive step for four instances $u l(h, i, j, k)$, $u l(h, i, 0, k), u l(0, i, 0, k)$, and $u l(0, i, 0,0)(h, i, j, k>0)$. We also show the inductive step for the (ctr) $)_{u l}$ rule. The translation of the other rules is trivial.

$$
\frac{\mathcal{R}, R^{i} v x, R^{k} u x, v: \Delta, u: \Delta^{\prime}, \Gamma}{\mathcal{R}, R^{h} w v, R^{j} w u, v: \Delta, u: \Delta^{\prime}, \Gamma} \frac{\mathcal{R}, R^{i} v x, R^{k} u x, v: \Delta, u: \Delta^{\prime}, \Gamma}{\frac{\mathcal{R}, R^{h} w v, R^{j} w u, R^{i} v x, R^{k} u x, v: \Delta, u: \Delta^{\prime}, \Gamma}{\mathcal{R}, R^{h} w v, R^{j} w u, v: \Delta, u: \Delta^{\prime}, \Gamma}}(\mathrm{wk})
$$


$\frac{\mathcal{R}, R^{i} v x, R^{k} u x, v: \Delta, u: \Delta^{\prime}, \Gamma}{\mathcal{R}, R^{h} w v, v: \Delta, w: \Delta^{\prime}, \Gamma}$

$\frac{\mathcal{R}, R^{i} v x, R^{k} u x, v: \Delta, u: \Delta^{\prime}, \Gamma}{\mathcal{R}, v: \Delta, v: \Delta^{\prime}, \Gamma}$

$$
\frac{\mathcal{R}, R^{i} v u, v: \Delta, u: \Delta^{\prime}, \Gamma}{\mathcal{R}, v: \Delta, v: \Delta^{\prime}, \Gamma}
$$

$$
\frac{\mathcal{R}, \mathcal{Q}, \hat{\mathcal{Q}}, \Delta, \hat{\Delta}, \Gamma}{\mathcal{R}, \mathcal{Q}, \Delta, \Gamma}
$$

$$
\frac{\frac{\mathcal{R}, R^{i} v x, R^{k} u x, v: \Delta, u: \Delta^{\prime}, \Gamma}{\mathcal{R}, R^{h} u v, R^{i} v x, R^{k} u x, v: \Delta, u: \Delta^{\prime}, \Gamma}}{\mathcal{R}, R^{h} u v, v: \Delta, u: \Delta^{\prime}, \Gamma} l(h, i, 0, k)
$$$$
\frac{\frac{\mathcal{R}, R^{i} v x, R^{k} u x, v: \Delta, u: \Delta^{\prime}, \Gamma}{\mathcal{R}, R^{i} v x, R^{k} v x, v: \Delta, v: \Delta^{\prime}, \Gamma}}{\mathcal{R}, v: \Delta, v: \Delta^{\prime}, \Gamma} l(0, i, 0, k)
$$$$
\begin{aligned}
& \frac{\mathcal{R}, R^{i} v u, v: \Delta, u: \Delta^{\prime}, \Gamma}{\mathcal{R}, R^{i} v v, v: \Delta, v: \Delta^{\prime}, \Gamma} \\
& \mathcal{R}, v: \Delta, v: \Delta^{\prime}, \Gamma
\end{aligned}
$$

$$
\frac{\mathcal{R}, \mathcal{Q}, \hat{\mathcal{Q}}, \Delta, \hat{\Delta}, \Gamma}{\mathcal{R}, \mathcal{Q}, \mathcal{Q}, \Delta, \Delta, \Gamma} \text { lem. } 6
$$

Example 9. The derivation of $x: \square \bar{p} \vee \diamond \diamond p$ in G3Kt $(U T)+u l(1,1,1,1)$ (see Example 8) can be transformed into a derivation in $\mathrm{G} 3 \mathrm{Kt}+l(1,1,1,1)$ as follows:

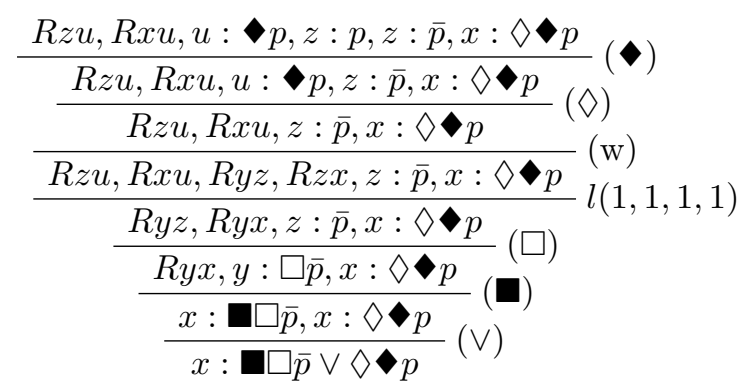

From G3Kt $+l(h, i, j, k)$ to $\mathrm{G} 3 \mathrm{Kt}(U T)+u l(h, i, j, k)$. Consider now the converse direction. Let $S$ be a finite set of Scott-Lemmon axioms and define

$$
\begin{aligned}
& S_{u l}=\left\{u l(h, i, j, k) \mid \boldsymbol{\square}^{h} \square^{j} \bar{p} \vee \diamond^{i} \diamond^{k} p \in S\right\} \\
& S_{l}=\left\{l(h, i, j, k) \mid \mathbf{\square}^{h} \square^{j} \bar{p} \vee \diamond^{i} \diamond^{k} p \in S\right\}
\end{aligned}
$$

Given a derivation $\delta$ in G3Kt $+S_{l}$, in general $\delta$ will not be a derivation in $\operatorname{GKt}(U T)+S_{u l}$ because some sequents in $\delta$ (possibly even the endsequent) may not be a $\mathrm{G} 3 \mathrm{Kt}(U T)$-sequent. A more meaningful question is: given a derivation of $x: A$ in G3Kt $+S_{l}$, is there a derivation of $x: A$ in G3Kt $(U T)+S_{u l}$ that is effectively related to $\delta$ ? The constraint that the new derivation is "effectively related" is crucial, for otherwise one could trivially relate $\delta$ with the derivation $\delta^{\prime}$ obtained from the following equivalence:

$$
\vdash_{\mathrm{G} 3 \mathrm{Kt}+S_{l}}^{\delta} x: A \text { iff } A \in \mathrm{Kt}+\boldsymbol{\square}^{h} \square^{j} \bar{p} \vee \diamond^{i} \diamond^{k} p \text { iff } \exists \delta^{\prime} . \vdash_{\mathrm{G} 3 \mathrm{Kt}(U T)+S_{u l}}^{\delta^{\prime}} x: A
$$


Although the phrase 'effectively related' has not been explicitly defined, what we envisage is a local (i.e. rule by rule) transformation on $\delta$, which is sensitive to its structure, that ultimately yields a $\mathrm{G} 3 \mathrm{Kt}(U T)+S_{u l}$ derivation of $x: A$. Notice that the $\mathrm{G} 3 \mathrm{Kt}(U T)+S_{u l}$ derivation obtained via the above argument is not sensitive to the input in the sense that any two $\mathrm{G} 3 \mathrm{Kt}+S_{l}$ derivations of $x: A$ would be mapped to the same $\mathrm{G} 3 \mathrm{Kt}(U T)+S_{u l}$ derivation.

In the boundary case for Kt when $S=S_{l}=S_{u l}=\emptyset$ we have the following result, which also establishes that G3Kt is an internal calculus with respect to derivations that end with a single formula.

Proposition 1. Every labelled derivation in G3Kt of $x: A$ is also a derivation in $\operatorname{GKK}(U T)$.

Proof. We argue by contradiction. Let $\delta$ be a derivation of $x: A$ in G3Kt and suppose there is a labelled sequent $\mathcal{R}, \Gamma$ in $\delta$ that is not a $\mathrm{G} 3 \mathrm{Kt}(U T)$-sequent. This means that the underlying graph of $\mathcal{R}$ is not a tree. If $\mathcal{R}$ is not connected, then by inspection of the rules of G3Kt, the underlying graph of every sequent below it (and hence $x: A$ ) would not be connected and this is a contradiction. On the other hand, if $\mathcal{R}$ is connected and its underlying graph is not a tree, then the underlying graph must contain a cycle. This follows from the fact that $\mathcal{R}$ is assumed connected, and the fact that any acyclic connected graph forms a tree. This means that there exist $x, y, w$ such that $\{R x w, R y w\} \subseteq \mathcal{R}$. By inspection of the rules of G3Kt, every sequent below $\mathcal{R}, \Gamma$ will contain this cycle contradicting the assumption that $x: A$ is the end sequent.

This argument does not work for extensions of G3Kt because the additional structural rules may be capable of removing cycles in the following sense: the underlying (i.e. undirected) graph of the premise might have a cycle yet the underlying graph of the conclusion might not (this was not the case for any rule in G3Kt). Indeed, consider the rule for transitivity:

$$
\frac{\mathcal{R}, R x y, R y z, R x z, \Gamma}{\mathcal{R}, R y z, R x z, \Gamma} \text { (Trans) }
$$

In a rule instance of (Trans), the underlying graph of the premise necessarily contains a cycle. However, it need not be the case that the underlying graph of the conclusion contains a cycle. As a consequence, a labelled derivation of $x: A$ in G3Kt + (Trans) may contain sequents whose underlying graph is not a tree. Such a derivation cannot be a derivation in any UT calculus.

It is tempting to replace (Trans) with its non-invertible form in order to remove the cycle. However the (ctr) rule seems not to be admissible in G3Kt + (Trans') which means that it needs to be included to ensure completeness. The simulation of the above rule instance in G3Kt $+(\mathrm{ctr})+\left(\right.$ Trans $\left.{ }^{\prime}\right)$ below right indicates that we have merely shifted the problem to a new setting.

$$
\frac{\mathcal{R}, R x z, \Gamma}{\mathcal{R}, R x y, R y z, \Gamma}\left(\text { Trans' }^{\prime}\right) \quad \frac{\mathcal{R}, R x y, R y z, R x z, \Gamma}{\mathcal{R}, R x y, R y z, R x y, R y z, \Gamma}\left(\begin{array}{l}
\text { Trans' } \\
\text { R }, \text { Rxy }, R y z, \Gamma
\end{array}\right.
$$


In summary: embedding the display calculus into the labelled calculus has yielded two seemingly distinct labelled calculi for the tense logics: $\mathrm{G} 3 \mathrm{~K} t+l(h, i, j, k)$ and $\mathrm{G} 3 \mathrm{Kt}(U T)+u l(h, i, j, k)$. Investigating the (im)possibility of a pointwise translation from the derivations in the former to the latter is an interesting problem which we defer to future work.

Acknowledgments. Work supported by the FWF projects: START Y544-N23 and I 2982.

\section{References}

1. N. D. Belnap, Jr. Display logic. J. Philos. Logic, 11(4):375-417, 1982.

2. P. Blackburn, M. de Rijke, and Y. Venema. Modal logic, volume 53 of Cambridge Tracts in Theoretical Computer Science. Cambridge University Press, 2001.

3. K. Brünnler. Deep sequent systems for modal logic. In Advances in modal logic. Vol. 6, pages 107-119. Coll. Publ., London, 2006.

4. A. Chagrov and M. Zakharyashchev. Modal companions of intermediate propositional logics. Studia Logica, 51(1):49-82, 1992.

5. A. Ciabattoni and R. Ramanayake. Power and limits of structural display rules. ACM Trans. Comput. Logic, 17(3):1-39, Feb. 2016.

6. R. Dyckhoff and S. Negri. Proof analysis in intermediate logics. Arch. Math. Log., 51(1-2):71-92, 2012.

7. R. Dyckhoff and S. Negri. Geometrization of first-order logic. The Bulletin of Symbolic Logic, 21:123-163, 2015.

8. M. Fitting. Proof methods for modal and intuitionistic logics, volume 169 of Synthese Library. D. Reidel Publishing Co., Dordrecht, 1983.

9. M. Fitting. Prefixed tableaus and nested sequents. Ann. Pure Appl. Logic, 163(3):291-313, 2012.

10. R. Goré, L. Postniece, and A. Tiu. On the correspondence between display postulates and deep inference in nested sequent calculi for tense logics. Log. Methods Comput. Sci., 7(2):2:8, 38, 2011.

11. R. Goré and R. Ramanayake. Labelled tree sequents, tree hypersequents and nested (deep) sequents. In Advances in modal logic, Vol. 9. College Publications, 2012.

12. G. Greco, M. Ma, A. Palmigiano, A. Tzimoulis, and Z. Zhao. Unified correspondence as a proof-theoretic tool. Journal of Logic and Computation, 2016.

13. R. Kashima. Cut-free sequent calculi for some tense logics. Studia Logica, 53(1):119-135, 1994.

14. M. Kracht. Power and weakness of the modal display calculus. In Proof theory of modal logic (Hamburg, 1993), volume 2 of Appl. Log. Ser., pages 93-121. Kluwer Acad. Publ., Dordrecht, 1996.

15. E. J. Lemmon and D. S. Scott. The 'Lemmon Notes': An Introduction to Modal Logic. Blackwell, Oxford, 1977.

16. G. Mints. Indexed systems of sequents and cut-elimination. J. Philos. Logic, 26(6):671-696, 1997.

17. S. Negri. Proof analysis in modal logic. J. Philos. Logic, 34(5-6):507-544, 2005.

18. R. Ramanayake. Inducing syntactic cut-elimination for indexed nested sequents. In Proceedings of IJCAR, pages 416-432, 2016.

19. G. Restall. Comparing modal sequent systems. http://consequently.org/ papers/comparingmodal.pdf. 
20. G. Restall and F. Poggiolesi. Interpreting and applying proof theory for modal logic. In G. Restall and G. Russell, editors, New Waves in Philosophical Logic, pages 39-62. 2012.

21. L. Viganò. Labelled non-classical logics. Kluwer Academic Publishers, Dordrecht, 2000. With a foreword by Dov M. Gabbay.

22. H. Wansing. Displaying modal logic, volume 3 of Trends in Logic-Studia Logica Library. Kluwer Academic Publishers, Dordrecht, 1998. 